\title{
Conceptual Analysis: technology, machine and robot
}

\author{
Aníbal Monasterio, Daniel López, Manuel Aparicio, Ricardo Morte, Txetxu Ausín and Mario Toboso
}

\begin{abstract}
In contrast to the hegemonic conceptual analysis from philosophy, we explore an alternative psychology based on perception and people's use of the words robot, machine and technology. To do this, we use computing tools to analyse information from Wikipedia and Google's search engine, which lets us envisage a digital paradigm in the understanding of robotics.
\end{abstract}

\section{INTRODUCTION}

$\mathrm{O}$ $\mathrm{NE}$ of the principle methods in philosophy is conceptual analysis. In its broadest sense, analysis consists of decomposing a phenomenon or problem into more basic and simple elements, and their explanation would consist in their reconstruction or synthesis, the opposite of analysis. This is the traditional decompositional version. But it is not the only one, nor the hegemonic. Another conception of analysis, used by, among others, Democritus and Descartes, is regressive analysis, which attempts to work back toward the fundamental principles in which something originates. But another concept of analysis exists, which can be seen in the work of Frege or Russell, known as interpretative or transformative, which says that for something to be analysed, first it must be transformed into a correct language for analysis, for example, a logical formal language [1].

Although for decades this way of understanding analysis has been one of the most-utilized and successful in philosophy, it has its limitations. Quine [2] presented a devastating critique of the same analytical/synthetic distinction that had dominated epistemology since the time of Kant. The theses laid out in this article, considered one of the most important in twentieth century philosophy, suggest that analytical truths and synthetic truths should be eliminated as they are circular notions that depend on synonymy. Quine's conclusions and the implications of his

This paper benefits from Grant Agreements 780073-INBOTS and 779982EXTEND

A. Monasterio is a Basque Government Postdoctoral Researcher, Bilbao, Spain (anibal.monasterio@ehu.eus).

D. López is a Predoctoral Researcher at the Department of Theoretical and Practical Philosophy in the Institute of Philosophy of the Spanish National Research Council, Madrid, Spain (daniel.lopez@csic.es).

M. Aparicio is an Associate Professor at the University of Murcia, Murcia, Spain (manuel.aparicio@um.es).

R. Morte is a Ph.D. Student at the University of Granada, Granada, Spain (ricardo63@autistici.org).

T. Ausín is a Tenured Scientist at the Department of Theoretical and Practical Philosophy in the Institute of Philosophy of the Spanish National Research Council, Madrid, Spain (txetxu.ausin@cchs.csic.es).

M. Toboso is a Tenured Scientist at the Department of Science, Technology and Society in the Institute of Philosophy of the Spanish National Research Council, Madrid, Spain (mario.toboso@csic.es).

They constitute the IFS-CSIC Research Group in the INBOTS and EXTEND projects. criticism of the analytical/synthetic distinction do not interest us here, but suffice to say that, if correct, the philosophical analysis method based on decomposing concepts and reducing them to their basic meaning would be doomed to failure. This is why we propose an interpretation of the concepts as psychological entities, and we explore the signifiers robot, machine and technology by means of information drawn from the internet. Finally, we highlight the phenomena we consider pertinent to the philosophic understanding of robotics.

\section{MATERIAL AND METHODS}

In addition to the traditional bibliographic review, we have imported tools from computational social science for this research with the goal of extracting relevant information on people's perceptions and interests regarding the words robot, machine and technology. The sources we chose were Wikipedia and Google. In the first case, we drew information from the API wordsimilarity.com (based on the word2vec model to process the semantic similarities of the Spanish words on Wikipedia) and the Pageviews Analysis tool (visits to the Wikipedia pages of these words between August 2016 and August 2018).

Before making a computerised analysis of the text, we must select the type of text to be included in our analysis. For our task of analysing the concepts robot, machine and technology, firstly we used a semantic text analytical tool (wordsimilarity.com) that allows data to be extracted from text corpus. This tool uses a semantic algorithm to process the similarities between different words. The API (application programming interface) of this tool uses Wikipedia pages as a database.

As we can see in Figures 1, 2 and 3, the concepts robot, machine and technology have the appearance of a Wittgensteinian family. The three concepts share analogous words, such as "tool," and many other similar ones. This fact leads us to believe that the use of these terms by people who are non-experts (usage we obtained indirectly from their searches on Wikipedia and with the Google search engine) is blurred. That is, one can observe a blurring of the conceptual border between robot, machine and technology. 


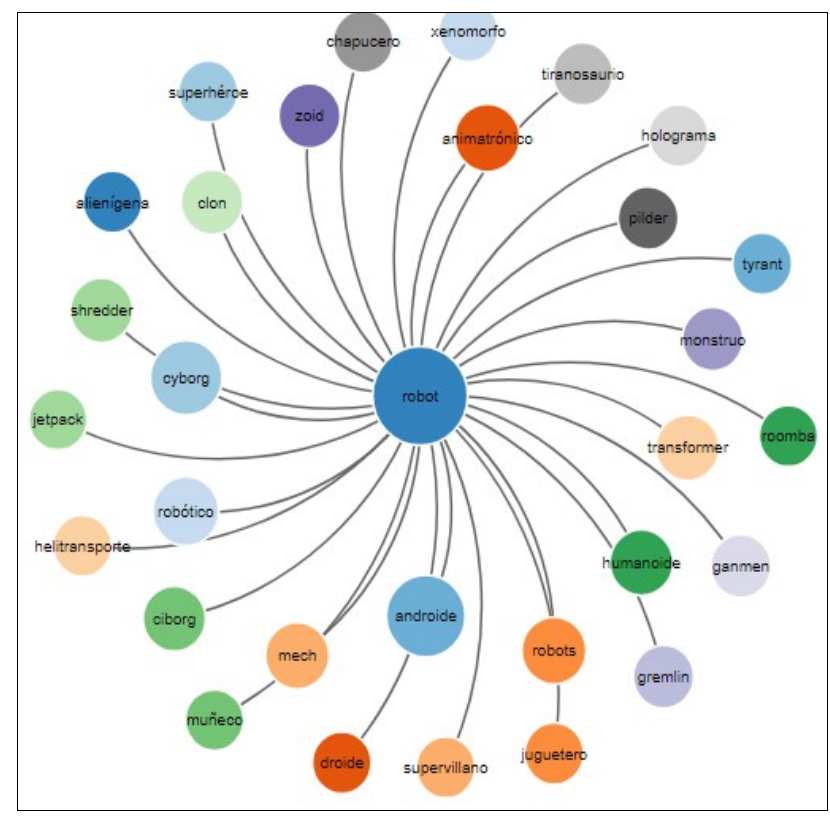

Figure 1. Thirty words similar or analogous to robot (in Spanish)

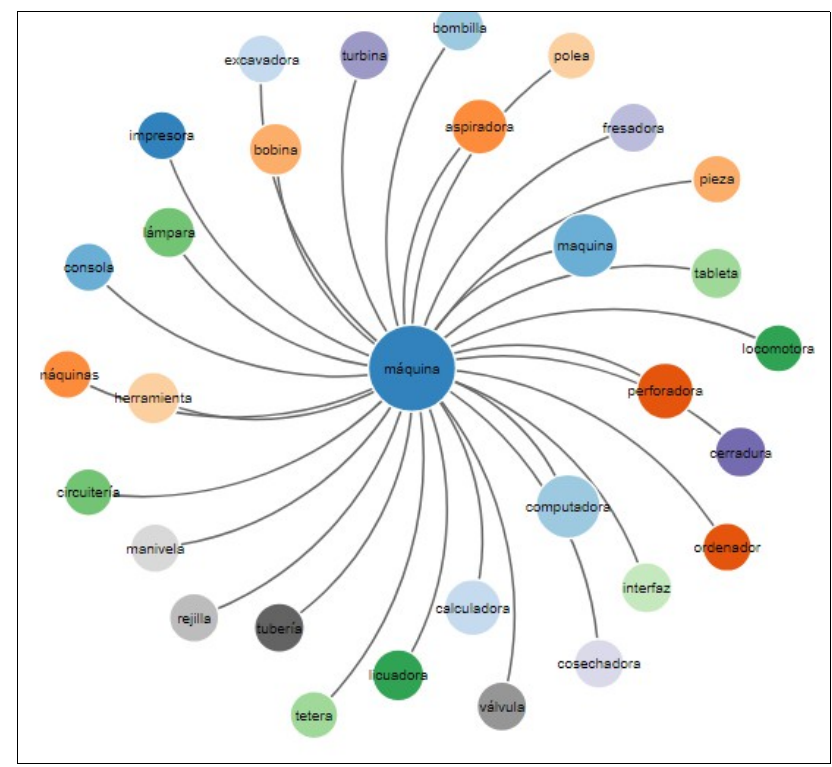

Figure 2. Thirty words similar or analogous to machine (in Spanish)

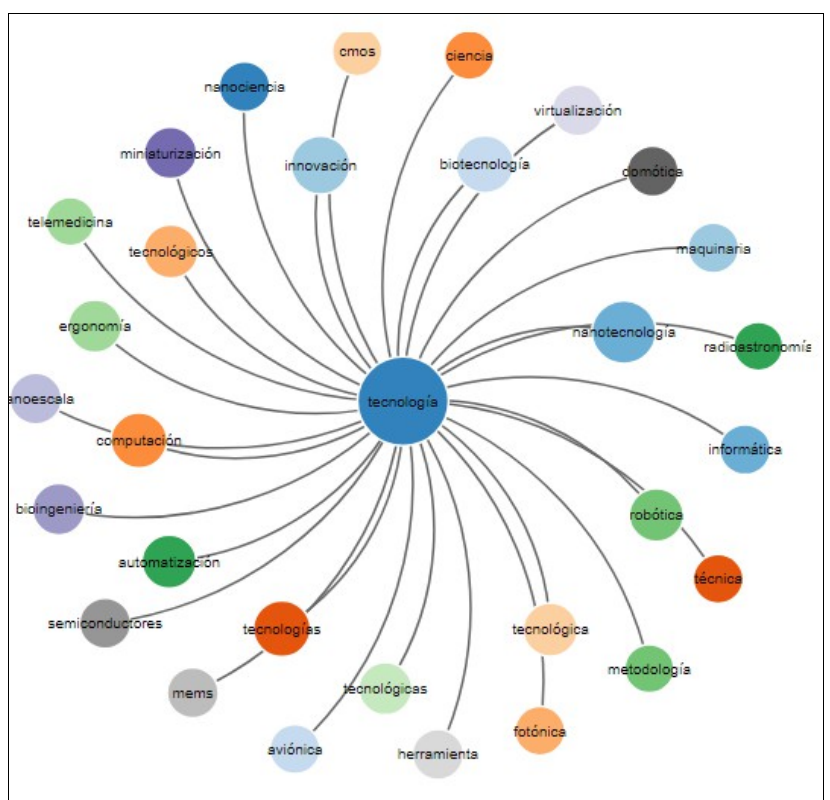

Figure 3. Thirty words similar or analogous to technology (in Spanish)

In the case of Google, we combined two tools imported from analytical methods for digital marketing: Keywords Everywhere and answerthepublic.com. In this case, we limited ourselves to the first 100 searches by volume for each term, and to the first ten questions formulated in natural language between August 2017 and August 2018. The choice is explained based on the following reasoning. Google's market share, in the case of Europe, reaches 93.32\% [3], which situates it as the main source of information on language usage. As compared to information published intentionally by an agent on the internet (whether on social media or a webpage), the data regarding user searches provides less-biased information about the curiosity, interests and concerns of users and/or citizens. In spite of the fact that at present asking questions of a search engine in natural language is still infrequent, the development and implementation of emerging technologies such as virtual assistants with AI (Siri, Google Assistant, Cortana, Alexa) as well as products intended for this interaction (Google Home or Amazon Echo) indicate that this way of accessing information and the classification criteria will become increasingly important. Lastly, extended mind theories demonstrate the relevance of these external elements in cognitive processes [4] and, as such, in the conceptualisation of the terms by the public. 


\section{Results}

From the perspective of concepts as psychological entities, a concept is retrieved from long-term memory in order to play a role in linguistic cognition and understanding. The concepts are psychological entities, not abstract entities [5]. In this sense, a concept of robot is a way of thinking about robots. Concepts are a body of information on individuals, classes, substances or events, what Machery [6] calls belief-like-states. Additionally, concepts are opaque: people do not have privileged access about the content of the concepts, and they may not be able to articulate it even though they have it. The distinction between what belongs to a concept and what does not is not semantic nor epistemological, it is psychological. It is not semantic because what belongs to a concept or what forms part of the background to the belief-like-states does not correspond to the analytic/synthetic distinction, but rather depends on what the world is like. And it is not epistemological because what belongs to a concept and what forms part of the background to the belief-like-states does not correspond to the a priori/a posteriori distinction.

The term robot comes from the Czech word robota, which came to mean servile work or slave. According to wordsimilarity.com, of the ten most similar words to robot, four are anthropomorphic (android, cyborg, transformer and humanoid). In Wikipedia, there is an average of approximately 2,300 daily hits on the entry "robot," being worthy of mention a peak of nearly 18,000 hits on December $27,2016$.

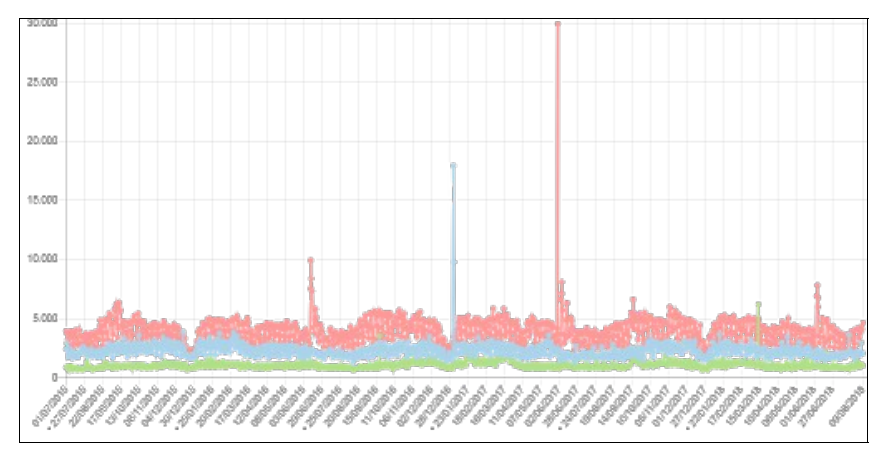

Figure 4. Analysis of visits to Wikipedia pages about robot, machine and technology from 1/07/2015 to 9/08/2018.

This anomaly may coincide with the interest stirred up by news about the human-controlled robot that took off on the North Korean border. Of the more than 4.8 million monthly searches done on Google that include the word robot, approximately $74 \%$ refer to entertainment (especially the programme War Robots, but also television series, films and videogames). Of this percentage, three-quarters are related to destruction or confrontations between robots and humans. Only $17 \%$ of searches refer to products available on the market, with the cleaning robot Roomba being the one in this group that arouses the most interest. Among the main questions in natural language made on Google's search engine, it is necessary to note that $50 \%$ refer to concerns such as "robots get citizenship?", "will robots take my job?" or "will robots replace humans?". (See Appendix)

The word machine comes from the Latin machina and the Greek $\mu \eta \chi \alpha v \eta$, and came to signify means or resource. According to wordsimilarity.com, the five words with the highest degree of similarity to machine are computer, calculator, drill, hoover and tool. In Wikipedia, there is an average of 1,100 daily visits to the entry with machine. We see a peak of visits on February 13, 2018 (around 6,200), which we speculate may have to do with a Gallup poll in the U.S. about worker's fears of being replaced by machines at their job.

Among the 4.3 million Google searches done monthly that have the word machine, it is worth noting that $39 \%$ refer to music groups or comics. The remaining $61 \%$ are divided between many different entities, highlighted by the search for merchandise (19\%), software to get likes on Facebook or Instagram (13\%), information related to machine learning $(10 \%)$ and how to do exercises with machines at the gym (7\%). The five most common questions in natural language are "when sewing machine was invented?", "are machine guns legal?", "how machine learning works?", "can machines think?", "why machine learning is important" (See APPENDIX)

The word technology comes from the Greek techné, which means art or trade, and logos, which means discourse or study. According to wordsimilarity.com, the five words with the highest degree of similarity to technology are nanotechnology, innovation, biotechnology, computing and automation. In Wikipedia there is an average of 4,000 monthly visits. We see a peak of 30,000 visits on May 24, 2017, the date on which the U.S. Federal Communications Commission (FCC) announced a proposal to eradicate net neutrality. Of the 1.2 million searches identified on Google, $53 \%$ refer to conceptual or educational information about technology. Twenty-five percent search for current news on its development and, finally, 15\% directly show concerns about the future. The five questions in natural language most asked on Google are: "how technology has changed our lives?", "why technology is important?", "why technology is bad?", "what technology wants"?, "why technology is good?" (See APPENDIX).

From the results obtained, we can draw a series of interesting correlations. First, the oft-mentioned influence from science-fiction is evident in the general conception of the word robot, alluding to an anthropomorphic being and surrounded by apocalyptic scenarios or in a destructive context. In this same sense, the questions in natural language as well as the peaks of hits on Wikipedia pages point to a generalized concern about the consequences of technological development. In respect to the term machine, we can surmise that its use has moved away from the traditional mechanical definition, with a considerable amount of inputs being related to software, machine learning and artistic phenomena. 


\section{Discussion}

Mechanical philosophy, which came to the modern world with Hobbes and Descartes, among others, would affirm that mechanisms (the causal structure behind the functioning of reality and nature) are no more than organized entities and/or activities that show the phenomenon to be explained. Furthermore, this metaphor (ex: computer as brain) has taken a roundtrip journey that has blurred its lines, becoming a place for the phenomenon to be explained in the same explanation. Floridi [7] already noted that the impact of ICTs on the human condition was due to, among other transformations, the blurring of distinctions between reality/virtuality and between human/machine/nature. In this same sense, we could be moving towards a new digital paradigm of robotics in which a mechanical structure capable of moving on three or more physical axes is not essential for robotics to be understood. Advances in computing have created a digital advantage, creating a heterochrony up to this day in mechanical, electrical and computing cogeneration. As such, when it comes to speaking about an "autonomous" or "intentional" interaction, all the digital creatures that, for example, Hernandez-Orallo [8] characterizes in his taxonomy of all minds would be included.

\section{CONCLUSIONS}

One of the conclusions highlighted in this approach is a blurring of the borders between what people understand for machine, robot and technology. If we add to this the softwareization of robotics (the change from a mechanistic paradigm of robotics to a digital paradigm of robotics), we have a holistic vision united with the general argument that the principle scientific metaphor to understanding reality is computational or algorithmic, which aims in the same direction as the aforementioned blurring of the borders between robot, machine and technology in public understanding.

\section{REFERENCES}

[1] M. Beaney, "Analysis," in The Stanford Encyclopedia of Philosophy, Summer 2018. E. N. Zalta, Ed. https://plato.stanford.edu/archives/sum2018/entries/analysis/; Metaphysics Research Lab, Stanford University, 2018.

[2] W. V. Quine, "Main Trends in Recent Philosophy: Two Dogmas of Empiricism," The Philosophical Review, vol. 60, no. 1, pp. 20-43, 1951.

[3] "Search Engine Market Share Europe", StatCounter Global Stats, 2018. [Online]. Available: http://gs.statcounter.com/search-enginemarket-share/all/europe [Accessed 22-Oct-2018].

[4] A. Clark and D. Chalmers, "The Extended Mind", Analysis, vol. 58, no. 1, pp. 7-19, 1998.

[5] E. Machery, "Concepts Are Not a Natural Kind," Philosophy of Science, vol. 72, no. 3, pp. 444-467, 2005.

[6] E. Machery, Philosophy Within Its Proper Bounds. Oxford University Press, 2017.

[7] L. Floridi, Ed., The Onlife Manifesto: Being Human in a Hyperconnected Era. Springer International Publishing, 2015.

[8] J. Hernández-Orallo, The Measure of All Minds. Cambridge Core, 2017.
Manual semantic classification of Google searches worldwide between August 2017 and August 2018 regarding robot in Figure 5. machine in Figure 6. and technology in Figure 7.

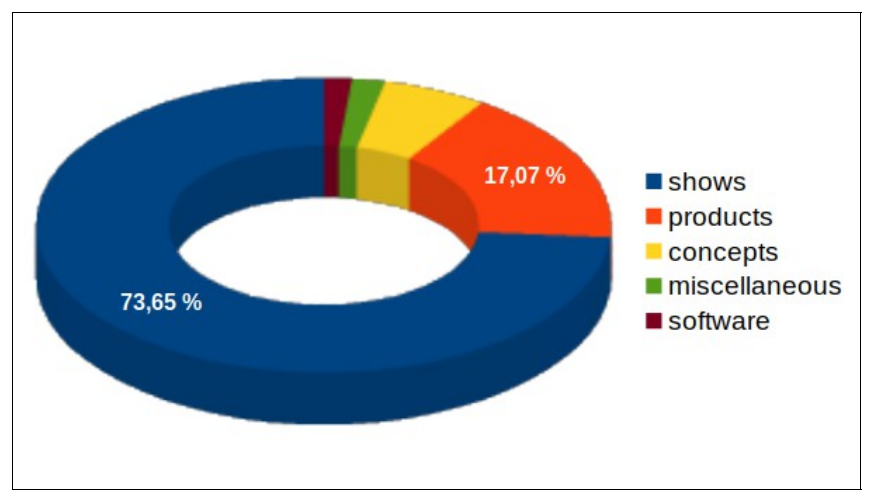

Figure 5.

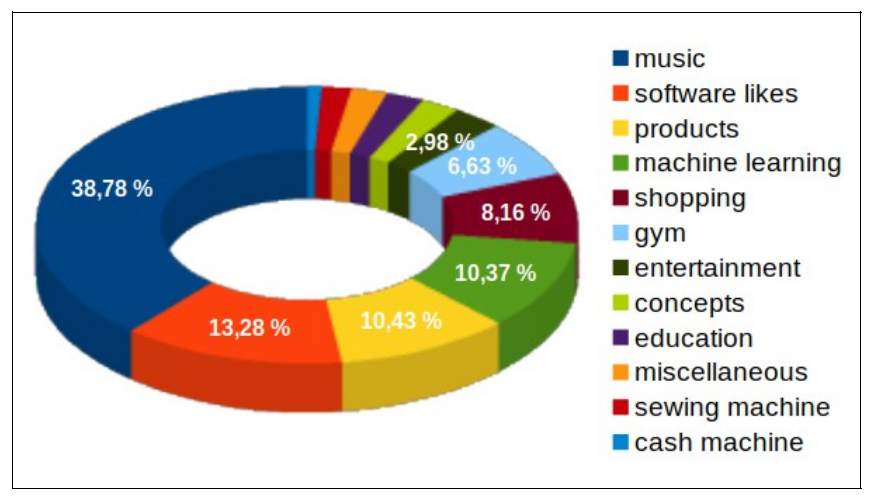

Figure 6.

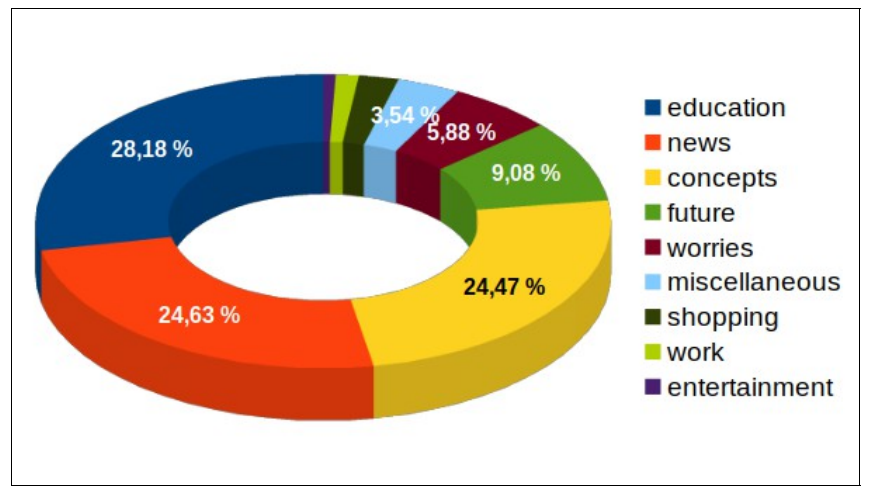

Figure 7. 